\title{
Spectral Changes in Cortical Surface Potentials during Motor Movement
}

\author{
Kai J. Miller, ${ }^{1}$ Eric C. Leuthardt, ${ }^{2}$ Gerwin Schalk, ${ }^{6}$ Rajesh P. N. Rao, ${ }^{3}$ Nicholas R. Anderson, ${ }^{7}$ Daniel W. Moran, ${ }^{7}$ \\ John W. Miller, ${ }^{4,5}$ and Jeffrey G. Ojemann ${ }^{2,5}$ \\ Departments of ${ }^{1}$ Physics, ${ }^{2}$ Neurological Surgery, ${ }^{3}$ Computer Science and Engineering, and ${ }^{4}$ Neurology and ${ }^{5}$ Harborview Regional Epilepsy Center, \\ University of Washington, Seattle, Washington 98195, ${ }^{6}$ Wadsworth Center, New York State Department of Health, Albany, New York 12201, and \\ ${ }^{7}$ Department of Biomedical Engineering, Washington University, St. Louis, Missouri 63130
}

In the first large study of its kind, we quantified changes in electrocorticographic signals associated with motor movement across 22 subjects with subdural electrode arrays placed for identification of seizure foci. Patients underwent a 5-7 d monitoring period with array placement, before seizure focus resection, and during this time they participated in the study. An interval-based motor-repetition task produced consistent and quantifiable spectral shifts that were mapped on a Talairach-standardized template cortex. Maps were created independently for a high-frequency band (HFB) $(76-100 \mathrm{~Hz})$ and a low-frequency band (LFB) $(8-32 \mathrm{~Hz})$ for several different movement modalities in each subject. The power in relevant electrodes consistently decreased in the LFB with movement, whereas the power in the HFB consistently increased. In addition, the HFB changes were more focal than the LFB changes. Sites of power changes corresponded to stereotactic locations in sensorimotor cortex and to the results of individual clinical electrical cortical mapping. Sensorimotor representation was found to be somatotopic, localized in stereotactic space to rolandic cortex, and typically followed the classic homunculus with limited extrarolandic representation.

Key words: electrocorticography (ECoG); motor; mapping; somatotopy; homunculus; cortical spectra

\section{Introduction}

Behavior-related changes in specific cortical spectral bands were described in animals as early as 1891, and similar task associated changes were demonstrated in humans brain potential measurements from the surface of the scalp (EEG) by 1929 (Berger, 1929; Brazier, 1961). Most subsequent studies of human cortical electrical behavior have focused on the spectral characteristics of EEG. The EEG frequency band ranges that have classically been associated with motor output are the $8-12 \mathrm{~Hz}$ (“ $\mu$ "), $18-26 \mathrm{~Hz}$ (“ $\beta$ ”), and everything $>30 \mathrm{~Hz}$ (" $\gamma$ ") (Wolpaw et al., 1991; Kostov and Polak, 2000; Pfurtscheller, 2000). The lower bands have been associated with thalamocortical circuits and typically decrease in amplitude in association with actual or imagined movements (Levine et al., 1999; Rohde et al., 2002; Pfurtscheller et al., 2003). Higher-frequency changes, in contrast, have been found to increase in amplitude with active or imagined motor movements

Received Sept. 7, 2006; revised Jan. 16, 2007; accepted Jan. 18, 2007.

K.J.M. was supported by the Poncin Award, the National Institutes of Health (NIH) Medical Scientist Training Program grant, and the Packard Award via R.P.N.R. K.J.M. and E.C.L. were supported by University of Washington Training Grant NS07144. J.G.0. was supported by NIH Grant NS41272. G.S. was supported by NIH Grants HD30146 and EB00856. R.P.N.R. was supported by National Science Foundation Grant 130705 and a Packard Fellowship. We thank neurologist Mark Holmes and the staff at Harborview Hospital in Seattle, Washington, and the neurologists and staff at Barnes-Jewish Hospital in St. Louis, Missouri, for their assistance. Discussions with Scott Makeig, Eric Edwards, and Pradeep Shenoy were very helpful in the process of analyzing and presenting the results contained in this paper.

Correspondence should be addressed to Kai J. Miller, University of Washington, Department of Physics, Box 351560, Seattle, WA 98195-1560. E-mail: kjmiller@u.washington.edu.

DOI:10.1523/JNEUROSCI.3886-06.2007

Copyright $\odot 2007$ Society for Neuroscience $\quad$ 0270-6474/07/272424-09\$15.00/0
(Crone et al., 1998; Pfurtscheller, 2001; Leuthardt et al., 2004) and may correlate with activity in local neuronal populations. The classic, although still debated (Nunez et al., 2001), inter pretation of changes in spectral amplitude, developed by Pfurtscheller and others (Pfurtscheller, 1981, 2000, 2001; Pfurtscheller et al., 2003, 2006), is that they reflect phase coherence of cortical circuits. With this interpretation came the suggestive labels event-related synchronization (ERS) and eventrelated desynchronization (ERD) and the postulate that spectral shifts in these potentials do not necessarily reflect differences in overall cortical computational activity but rather that they reflect changes in population coherence.

The present study aims to identify aspects of functional organization in the brain, using spectral shifts in the electrocorticographic (EcoG) array, when the state of motor cortex is changed in a supervised, interval-based, and repetitive manner. The high fidelity of ECoG signals has made them a source of interest for functional brain mapping, and this study is not the first to use them in this capacity. Crone and colleagues (Crone, 1998, 2000; Crone et al., 1998) demonstrated somatotopy in several (five) individuals using a paradigm with tonic muscle contraction. Tonic movement produced transient suppression at low frequencies and transient potentiation at high frequencies. These studies found high-frequency changes to be more spatially focal than low-frequency changes, and their methods reported variable somatotopy, with much involvement outside of classically described sensorimotor cortex. Pfurtscheller et al. (2003), using self-paced movement, reaffirmed the finding of spatially broad $\mu$ 
Table 1. Information for subjects who participated in the study

\begin{tabular}{|c|c|c|c|c|c|c|}
\hline Patient & Age & Sex & Hand & Coginitive capacity & Grid location & Seizure focus \\
\hline 1 & 31 & $\mathrm{~F}$ & $\mathrm{R}$ & Normal $^{a}$ & R frontotemporal & Rinsula \\
\hline 2 & 24 & $\mathrm{~F}$ & $\mathrm{R}$ & Normal & R frontotemporal & R orbitofrontal and tempora \\
\hline 3 & 21 & M & $\mathrm{R}$ & Normal & R frontoparietal & R parietal \\
\hline 4 & 38 & M & $\mathrm{R}$ & Borderline $(I 0,70)$ & R frontal & R frontal \\
\hline 5 & 48 & M & $\mathrm{R}$ & Borderline $(I Q, 82)$ & R temporal-parietal-occipital & R temporal-occipital \\
\hline 6 & 39 & $\mathrm{~F}$ & $\mathrm{R}$ & Normal & R frontal & R frontal \\
\hline 7 & 21 & M & $\mathrm{R}$ & Borderline $(I 0,76)$ & R frontotemporal & R frontal \\
\hline 8 & 23 & M & $\mathrm{R}$ & Normal & L frontotemporal & L temporal \\
\hline 9 & 22 & $\mathrm{~F}$ & $\mathrm{R}$ & Normal & L frontal & L frontal \\
\hline 10 & 35 & $\mathrm{~F}$ & L & Normal & L frontotemporal-parietal & L temporal \\
\hline 11 & 33 & M & $\mathrm{L}$ & Mildly impaired & L frontotemporal-parietal & L frontotemporal-parietal \\
\hline 12 & 23 & M & $\mathrm{R}$ & Normal $^{b}$ & L frontotemporal & L temporal \\
\hline 13 & 18 & $\mathrm{~F}$ & $\mathrm{R}$ & Normal & L frontal & L frontal \\
\hline 14 & 25 & $M^{\prime}$ & $\mathrm{R}$ & Normal & L temporoparietal & L temporal \\
\hline 15 & 41 & M & $\mathrm{L}$ & Normal & L frontotemporal & L temporal \\
\hline 16 & 25 & M & $\mathrm{L}$ & Normal & L frontal & L interhemispheric \\
\hline 17 & 19 & M & $\mathrm{R}$ & Borderline $(I 0,85)$ & B frontotemporal strips & R temporal and Insular \\
\hline 18 & 26 & M & $\mathrm{R}$ & Normal & L parietal-temporal-occipital & L ant occipital/post tempora \\
\hline 19 & 46 & $\mathrm{~F}$ & $\mathrm{~L}$ & Normal & L frontal & L frontal \\
\hline 20 & 26 & $\mathrm{~F}$ & $\mathrm{R}$ & Normal & R frontal & R frontal \\
\hline 21 & 32 & M & $\mathrm{R}$ & Normal & L frontotemporal-parietal & L temporal \\
\hline 22 & 27 & $\mathrm{~F}$ & $\mathrm{R}$ & Normal & L frontotemporal-parietal & L frontal \\
\hline
\end{tabular}

$M$, Male; F, female; L, left; $R$, right; IQ, intelligence quotient; ant, anterior; post, posterior.

${ }^{a}$ Congenital absence of left arm.

${ }^{b}$ Previous L ATL.

and $\beta$ ERD in ECoG, with more focused high gamma ERS. Loose somatotopy, unbound by rigid homuncular representation, was also reported in ECoG by Marsden et al. (2000), Hoshida and Sakaki (2003), and Branco et al. (2003).

Consistent with previous studies, we find that in the lowfrequency regimen $(<32 \mathrm{~Hz})$, distinct spectral peaks, which are present in resting intervals, decohere during movement intervals. At higher frequencies, we found broad spectral increase with movement that might represent increased neuronal activity across the entire neuronal ensemble measured by a single electrode, reflecting the summation en masse of many superimposed local field potentials (LFPs), each of which displays a shift in its own power law-type noise spectra (Beggs and Plenz, 2003, 2004).

By studying a large number of patients (22 subjects) and using stereotactic normalization and averaging techniques, our results help provide a generalizable, in-depth, and comprehensive characterization of low- and high-frequency changes in and around sensorimotor cortex during movement.

\section{Materials and Methods \\ Subjects}

Simple motor tasks were studied in 22 patients ( 10 females, $18-48$ years of age) (Table 1) who had undergone placement of intracranial electrode arrays to localize seizure foci before surgical treatment of medically refractory epilepsy. The arrays were typically placed for $5-7 \mathrm{~d}$ with the location of the electrodes and duration of implantation determined independently by clinical criteria alone. Experiments were performed at two institutions, University of Washington (UW) and Washington University in St. Louis (WashU). Patients underwent craniotomy for electrode placement and were typically studied $4-6 \mathrm{~d}$ after electrode placement to allow for recovery from the surgery. Only patients with some peri-rolandic coverage were included. Subjects gave informed consent for participation in a manner approved independently by the respective Institutional Review Boards.

\section{Recordings}

The platinum electrode arrays (Ad-Tech, Racine, WI) were typically configured as linear strips or $8 \times 8$ electrode arrays. The electrodes had $4 \mathrm{~mm}$ diameter (2.3 mm exposed), $1 \mathrm{~cm}$ interelectrode distance, and were embedded in SILASTIC. The recording system differed in technical features between the two centers. At UW, the ECoG signals were split into two identical sets. One set was fed into the clinical EEG system, and the other set was recorded with Synamps2 (Neuroscan, El Paso, TX) biosignal amplifiers. At WashU, a clinical telemetry system (XLTEK, Oakville, Ontario, Canada) was used, and the signal was acquired via the internal network. ECoG signals were then acquired from the Neuroscan or XLTEK system using the general-purpose software BCI2000 (Schalk et al., 2004). At UW, the sampling frequency was $1000 \mathrm{~Hz}$. At WashU, the sampling rate was 300,500 , or $600 \mathrm{~Hz}$. The signals were bandpass filtered from 0.15 or 0.3 to $200 \mathrm{~Hz}$ at both institutions. In this way, the $8-100 \mathrm{~Hz}$ spectral regions examined in this study are outside of any filtering ranges and below any relevant Nyquist frequency.

\section{Tasks}

Motor tasks were performed as part of a prescreening task for closed-loop brain-computer interface feedback experiments (Leuthardt et al., 2004, 2006). Patients performed simple, repetitive, motor tasks of hand (synchronous flexion and extension of all fingers, i.e., clenching and releasing a fist at a self-paced rate of $\sim 1 \mathrm{~Hz}$ ) or tongue (opening of mouth with protrusion and retraction of the tongue, i.e., sticking the tongue in and out, also at $1 \mathrm{~Hz}$ ). These movements were performed in an interval-based manner, alternating between movement and rest, and the side of movement was always contralateral to the side of cortical grid placement. Additional movement types were flexion and extension of a given finger, audible repetition of the word "move," pursing and unpursing of the lips, and adduction and abduction at the hip, shoulder, and ankle. There were between 30 and 75 cue presentations for each movement modality. Cues for motor movement were delivered visually in a $10 \times 10 \mathrm{~cm}$ presentation window at a distance of $75-100 \mathrm{~cm}$ from patient. Visual cues were presented using the BCI 2000 program (Schalk et al., 2004) in one of three ways: a colored box, a fixation point (" $\mathrm{x}$ ") cuing a specific motor movement, or a written word indicating the specific body part to be moved (typically, multiple movement types were interleaved in each experimental run). Stimuli were presented for $2 \mathrm{~s}$ (patients $1,2,3,5,8$, 17) or $3 \mathrm{~s}$ (all other patients), followed by rest intervals (indicated by a blank screen) of the same length. The patients were instructed to perform repetitive, self-paced motor movement, alternating with rest intervals of 

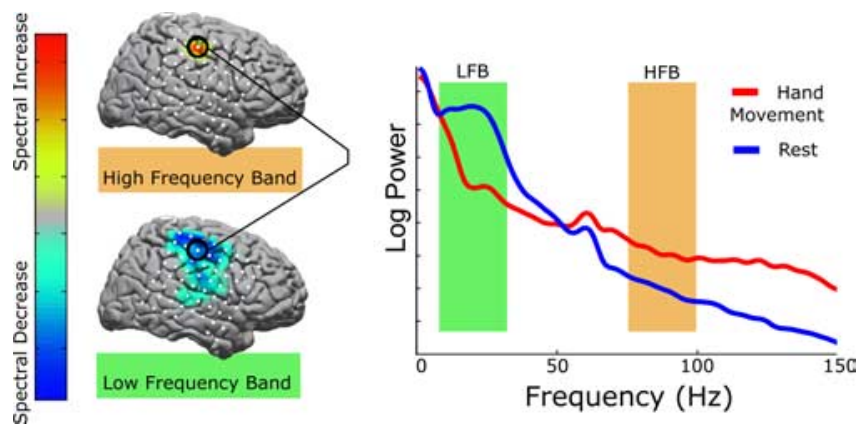

Figure 1. Demonstration of the analysis technique for a hand movement task in patient 5. The shaded areas in the spectral plot illustrate the bands used for analysis. The green shaded region is from 8 to $32 \mathrm{~Hz}$ (LFB), and the orange shaded region is from 76 to $100 \mathrm{~Hz}$ (HFB). The top cortical map shows the activation distribution for the HFB (here, reflecting an increase in spectral power with movement). The bottom map, for the LFB, shows a decrease in spectral power with movement over a broader set of electrodes. The color bar indicates the scale used for all spectral maps: blues reflect spectral decrease, and red-yellow reflects spectral increase. Gray indicates no change. All cortical maps are scaled to the maximum increase or decrease; thus, there is no relevant absolute scale. Electrode locations are shown in white.

the same length (indicated by the absence of the cuing target). Repetitive motion, rather than tonic contraction, was intended to accentuate the spectral shift during each interval, because attenuation of $\alpha(6-12 \mathrm{~Hz})$ and $\beta(18-26 \mathrm{~Hz})$ decrease (Crone et al., 1998) and $\gamma(>30 \mathrm{~Hz})$ increase (Crone et al., 1998) has been reported during tonic contraction. Multiple movement types were interleaved in each experimental run.

\section{Signal analysis}

All ECoG data sets were re-referenced with respect to the common average. The 0.5-2.0 s epoch was used for analysis within each interval of activity or rest, regardless of sampling frequency or cue duration. The power spectral density of each epoch was calculated every $1 \mathrm{~Hz}$ from 1 to $150 \mathrm{~Hz}$ using a Fast Fourier Transform using $0.25 \mathrm{~s}$ windows with an overlap of $0.1 \mathrm{~s}$, and was averaged throughout each epoch. A Hann window (Nuttall, 1981) was imposed on each data window to attenuate edge effects. The number of rest epochs was randomly downsampled so that the number of rest epochs and the number of active epochs of each type were the same, for balanced statistical comparisons. The power at each frequency (in $1 \mathrm{~Hz}$ intervals) for each epoch was normalized with respect to the mean power at that frequency across all epochs in the run from which it came. We then calculated the sum of all normalized power values in two $25 \mathrm{~Hz}$ bands. Normalization was deemed necessary because the "typical" ECoG spectrum resembles a power law (Fig. 1), and thus the changes at the lower end of either band would dominate the analysis procedure without it. The approach of taking wide-frequency ranges avoided chauvinism toward any specific frequency and accentuated the effect of broad spectral changes (Figs. 1, 2).

Low-frequency band $(8-32 \mathrm{~Hz})$. This band spans is the classic " $\mu-\beta$ " region of the spectra that typically exhibits amplitude decrease with motor movement. The fact that a peak in this region dissolves has led to the term ERD. Because it is a peak that disappears, it suggests dissolution of native timescale(s). These timescales may correspond to native thalamocortical timescales (Lopez da Silva, 1991; Neidermeyer, 1999) or perhaps some other inhibitory circuit that is activated when the brain region of interest is functionally engaged. This band is also picked to flank the classic range that EEG studies have focused on.

High-frequency band $(76-100 \mathrm{~Hz})$. This range is chosen to be representative of the spectrally broad power increase that accompanies movement in relevant, focal brain areas. This particular interval was chosen because it lies within this broad increase, avoids $60 \mathrm{~Hz}$ contamination, and matches the $25 \mathrm{~Hz}$ width of the low-frequency band (LFB). Although this interval was chosen arbitrarily and does not capture the entire broadspectral change, supplemental Figures S6 and S7 (available at www.jneurosci.org as supplemental material) demonstrate how our results would be very much the same if we had picked a different frequency band within this broad spectral increase for our high-frequency band (HFB) analysis.
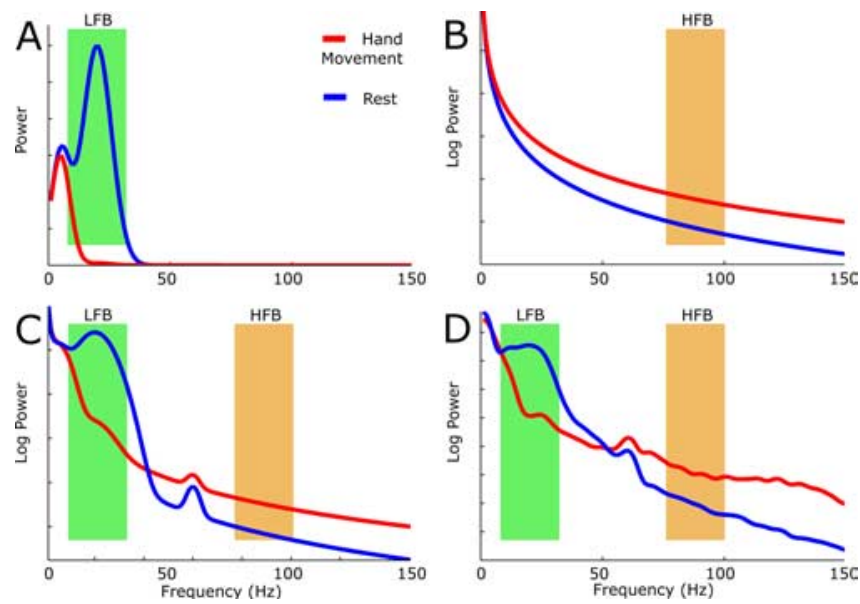

Figure 2. The power spectra for the motor task (red) and rest intervals (blue) illustrate the rationale and tentative hypothesis behind the choice of spectral bands (HFB and LFB) for analysis. $\boldsymbol{A}-\boldsymbol{D}$, The first three plots $(\boldsymbol{A}-\boldsymbol{C})$ are elements of artificial schema, and the fourth $(\boldsymbol{D})$ is actual data (the same as in Fig. 2). $\boldsymbol{A}$, Modeling the ERD. In the rest condition, it is proposed that native timescales of 200 and $50 \mathrm{~ms}$, generating spectral peaks based at 5 and $20 \mathrm{~Hz}$, arise from cortical regulation by thalamus and/or other structures. With motor activation, one or more of these timescales decohere ("desynchronize"). Note that the rest period here does not necessarily reflect a baseline state, but the rest interval between actions, and may therefore be more coherent ("synchronized") than a true baseline state. The schematic assumes $99 \%$ decoherence of the $20 \mathrm{~Hz}$ spectral peak with respect to the resting state. The green band reflects the analysis band (LFB) used to capture this effect. $\boldsymbol{B}$, Modeling the broad spectral increase. In this model, a power law spectral shift from, for example, $1 / f^{2.3}$ in the rest state to $1 / f^{2}$ in the motor state, is diagrammed. This broad increase could also be a result of white noise addition to the data (a uniform shift upward). The orange band (HFB) is chosen to capture this shift because it is away from masking by discrete, native, timescales at lower frequencies (as in $\boldsymbol{A}$ ) and noise contamination at 60 $\mathrm{Hz}$. $\boldsymbol{C}$, Superposition of the spectra in $\boldsymbol{A}$ and $\boldsymbol{B}$, with the addition of $60 \mathrm{~Hz}$ noise (the same amount to each state). The orange and green bands are as before. $\boldsymbol{D}$, Actual shift seen. This spectrum is the same as in Figure 2. Note the similarity to the modeled spectrum (C).

For every electrode and movement type, we calculated a high and low activation weight by comparing the distributions of HFB and LFB values for each movement type with the corresponding rest distributions. Each such weight $(A)$ was a signed squared cross-correlation value, a measure of how much of the variance in power across both movement and rest epochs was accounted for by the difference in the mean power between movement and rest epochs, as detailed in Equation 1. We calculated the $p$ values associated with power in these normalized bands using a balanced, unpaired $t$ test with the normalized HFB and LFB power for a given movement type compared with rest. For each subject, the calculated $p$ value was Bonferroni corrected (Bonferroni, 1936) for the number of channels.

$$
A_{m r}=\frac{(\bar{m}-\bar{r})^{3}}{|\bar{m}-\bar{r}| \sigma_{m \cup r}^{2}} \frac{N_{m} N_{r}}{N_{m \cup r}^{2}} .
$$

This formula was used to generate the factor that scales each Gaussian kernel at each electrode location. $m$ denotes the distribution of movement interval weights, and $r$ denotes the distribution of rest interval weights, with $m 200 r$ denoting the union of the two distributions. $N$ is the number of elements in each distribution, $\sigma$ is the $\mathrm{SD}$, and a bar denotes distribution mean. (This equation is the square of the cross-correlation coefficient, with a sign added to indicate whether the change is an increase or decrease.)

\section{Electrode localization}

As in other studies (Zacks et al., 2003, 2004), the electrode placement was documented clinically by its relationship to skull table and landmarks from the sagittal (lateral) and coronal (anterior-posterior) skull x rays. We used these $\mathrm{x}$ rays to derive normalized Talairach coordinates for the each of the electrode arrays using the LOC (location on cortex) localiza- 

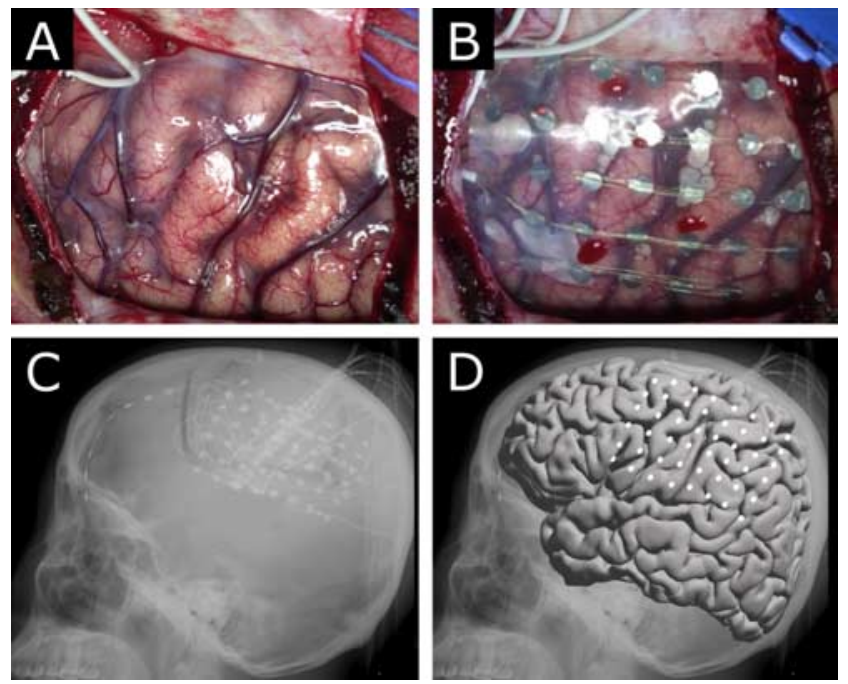

Figure 3. Grid placement and representation on a brain template. $A, B$, The brain is exposed $(\boldsymbol{A})$ and electrodes are placed in the subdural space $(\boldsymbol{B})$ to identify seizure onset and map cortex for clinical purposes before resection of epileptic focus. $C, D$, The electrodes are localized with a lateral skull $x$ ray (Miller et al., 2007) (C), and positions transformed to a standardized template (D). White dots represent the center of the electrode location in the standardized Talairach coordinate system (Talairach and Tournoux, 1988).

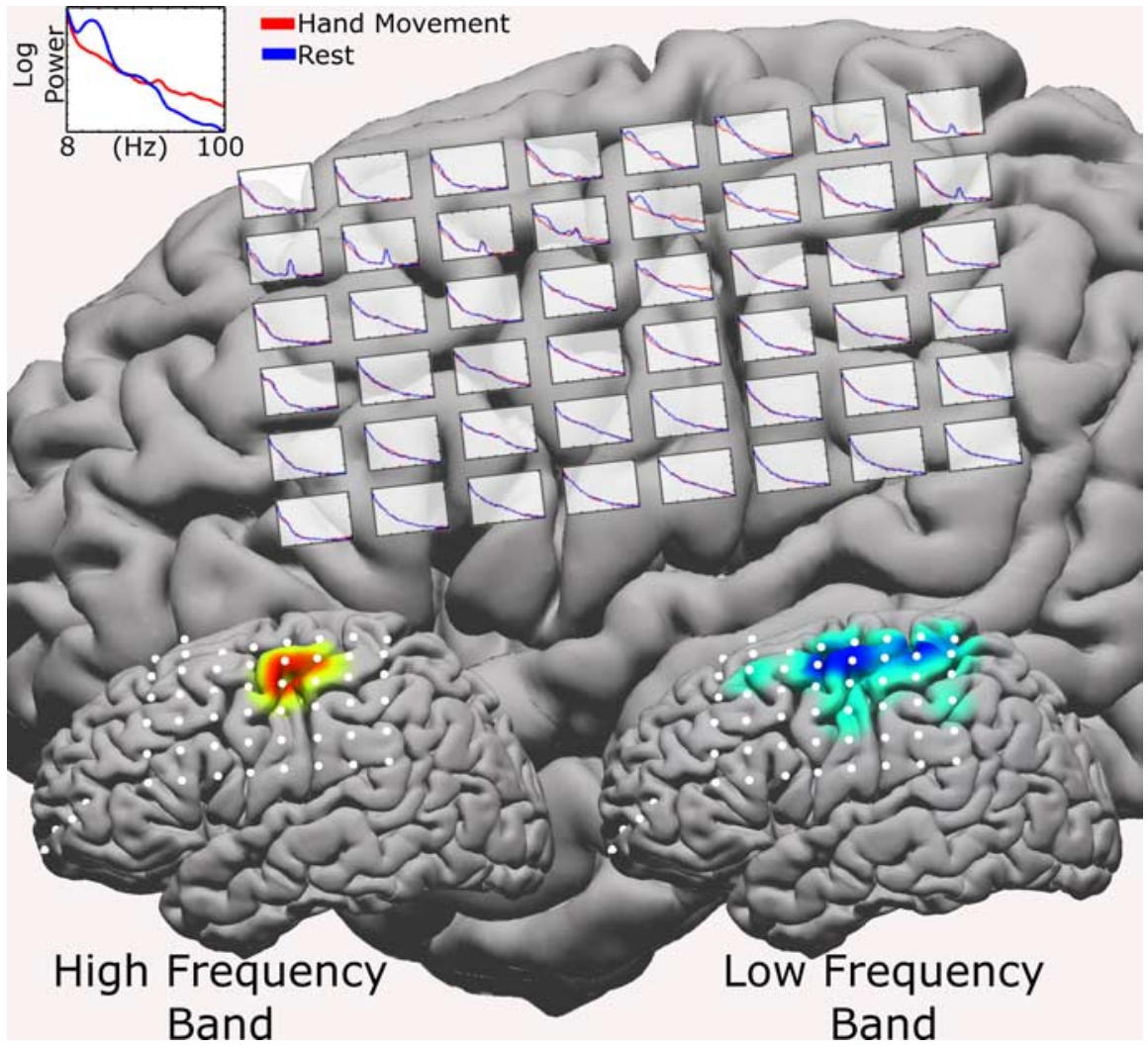

Figure 4. Spectra superimposed on the brain over each electrode for patient $J$ C during a hand movement task (red curves) and resting interval (blue curves). The frequency range (see top left inset) is $8-100 \mathrm{~Hz}$. Note that $60 \mathrm{~Hz}$ noise varies across electrodes (the two chosen frequency bands are away from this artifact). The bottom cortical insets reflect the generated cortical activation maps, with the HFB map on the left cortex and the LFB map on the right. Note that, as expected by the bottom insets, the most dramatic effects in the individual spectra are seen in the top, middle portions of the grid.

tion package (Miller et al., 2007), which is itself based in part on the manual procedure described by Fox et al. (1985) (Fig. 3). Skull landmarks were used to determine brain dimension for standardization, and the position of the radio-opaque electrodes with respect to these skull land-

\section{Results}

marks allowed for calculation of the position of each electrode in standardized (Talairach and Tournoux, 1988) coordinates. In areas of low convexity with respect to the lateral $\mathrm{x}$ ray (such as rolandic cortex) only an anterior-posterior ordinate and a superior-inferior ordinate were needed because the third ordinate could be uniquely determined by the convex surface of a template brain. Electrodes in areas of high convexity with respect to the lateral $\mathrm{x}$ ray required the use of the AP $\mathrm{x}$ ray to determine the third ordinate. Localizing electrodes based on their relationship to venous or gyral anatomy (Modayur et al., 1997) would be subject specific and not generalizable, but interpretation of activations with respect to cortical gyri would be straightforward. The method we used of plotting these standardized locations to the AFNIMontreal Neurological Institute template brain (Collins et al., 1995), in contrast, had the advantage of straightforward generalizability across subjects. It did suffer from the drawback that activation locations were calculated in this standardized space, without respect to gyri or other known cortical structures, and any relationship of activations to cortical structures in the template cortex must be interpreted with this in mind.

\section{Template brain mapping}

For template brain mapping, we included only electrodes with changes in power significant at the 0.05 Bonferroni-corrected level. We created activation maps independently for the HFB and LFB in each patient, for each movement type. We created these maps by linear superposition of spherical Gaussian kernels $(\mathrm{SD}=5 \mathrm{~mm}$ ) centered at the location of each electrode. The kernels were scaled by the activation weight associated with the given electrode and modality (Fig. 4) and interpolated at each point in a template brain. Maps for tongue and hand movement were averaged using linear summation of activation brain maps across all subjects for each task. Whereas this simple linear superposition may cause areas with denser electrode coverage to artificially appear to have increased activation compared with areas that have similar activation but less coverage across which to summate, our present method did not suffer from the types of spurious artifact that can accompany other methods. (For example, scaling of individual maps before superposition will skew toward peripheral activation of subjects with poor coverage but some significant activation, and point-by-point interpolation must account for single patients with anomalous activation weights.) For complete visualization of the analysis technique, please see supplemental Figure S1, available at www.jneurosci.org as supplemental material.

\section{Cortical stimulation mapping}

In seven patients, cortical stimulation mapping (Ojemann, 1982; Ojemann et al., 1989; Chitoku et al., 2001) of motor cortex was performed for clinical purposes. Each such stimulation patient underwent stimulation mapping to identify motor and speech cortices as part of his/her clinical care. In this mapping, 5-10 mA square wave current pulses (1 $\mathrm{ms}$ in length) were passed through paired electrodes for up to $3 \mathrm{~s}$ (less if a response is evoked) to induce sensation and/or evoke motor responses (see Fig. 9).

Activation maps for hand and tongue movement are shown in Figures 5 and 6 for all individuals and for the group average from each hemisphere. Supplemental material characterizes this for 
other movement types (supplemental Figs. S2, S3, available at www.jneurosci.org as supplemental material). A highly generalized response was found for motor tasks across individual subjects. Somatotopically defined decreases in low-frequency band (LFB) and increases in highfrequency band (HFB) were a consistent, significant feature in most subjects. These changes typically extended across sensorimotor cortex for the LFB and were somewhat more spatially specific for the HFB. Consistent with previous literature, spectral decreases were narrowly focused in the traditional $\mu / \beta$ bands, but increases were distributed over the entire range of higher frequencies. Over sensorimotor cortex, a typical " $\mu$ " peak was seen at $\sim 20-30 \mathrm{~Hz}$ that reliably decohered with activity. Along with this "lower" frequency change, a broad, very high-frequency increase in power is seen and, in our data, seems localized to sensorimotor cortex (Fig. 4, supplemental Fig. S4, available at www.jneurosci.org as supplemental material). Typically, the rest and active spectra intersected between 40 and $50 \mathrm{~Hz}$ in relevant electrodes (Fig. 4, supplemental Fig. S4, available at www.jneurosci.org as supplemental material). These findings were highly consistent across subjects. For example, we found statistically significant LFB decreases in 17 of 18 subjects with hand tasks and 14 of 16 with tongue tasks and HFB increases in 15 of 18 subjects with hand tasks and 14 of 16 with tongue tasks (Figs. 5, 6), and most of those in which significant changes were not found had less extensive rolandic coverage.

Taken both individually and on average (in collated, normalized, subject data), electrocorticographic signal changes were consistent with known localization of sensorimotor cortex. Figures 5, 6, and $7 \mathrm{dem}$ onstrate the individual and average activation maps for both HFB and LFB. The average activation locations for the HFB and the LFB [hand: high $( \pm 47,-15,50)$, low $( \pm 50,-15,50)$; tongue: high $( \pm 60,-6,31)$, low $( \pm 58,-6,39)$; in Talairach-normalized coordinates] fall within stereotactically defined Brodmann area 4 for hand and tongue. The somatotopy of these two motor areas matched the established homuncular distribution, and the distributions of spectral changes were anatomically distinct for hand and tongue movement, as shown in Figure 7 (spatial separability was significant, with an associated $p$ value of $<0.001$ for both HFB and LFB, using a Bonferroni corrected, unpaired, $t$ test of all electrodes, which showed a significant change for the movement of concern, with locations from all individuals concatenated).

Analysis of many movement modalities in several of the individuals (Fig. 8) showed both loose somatotopy and pericentral localization. Supplementary motor activation was seen in a single subject with medial coverage (Fig. $8 \mathrm{C}$ ). Areas of activation for different modalities overlapped, and the overlap was more extensive in the LFB than the HFB.

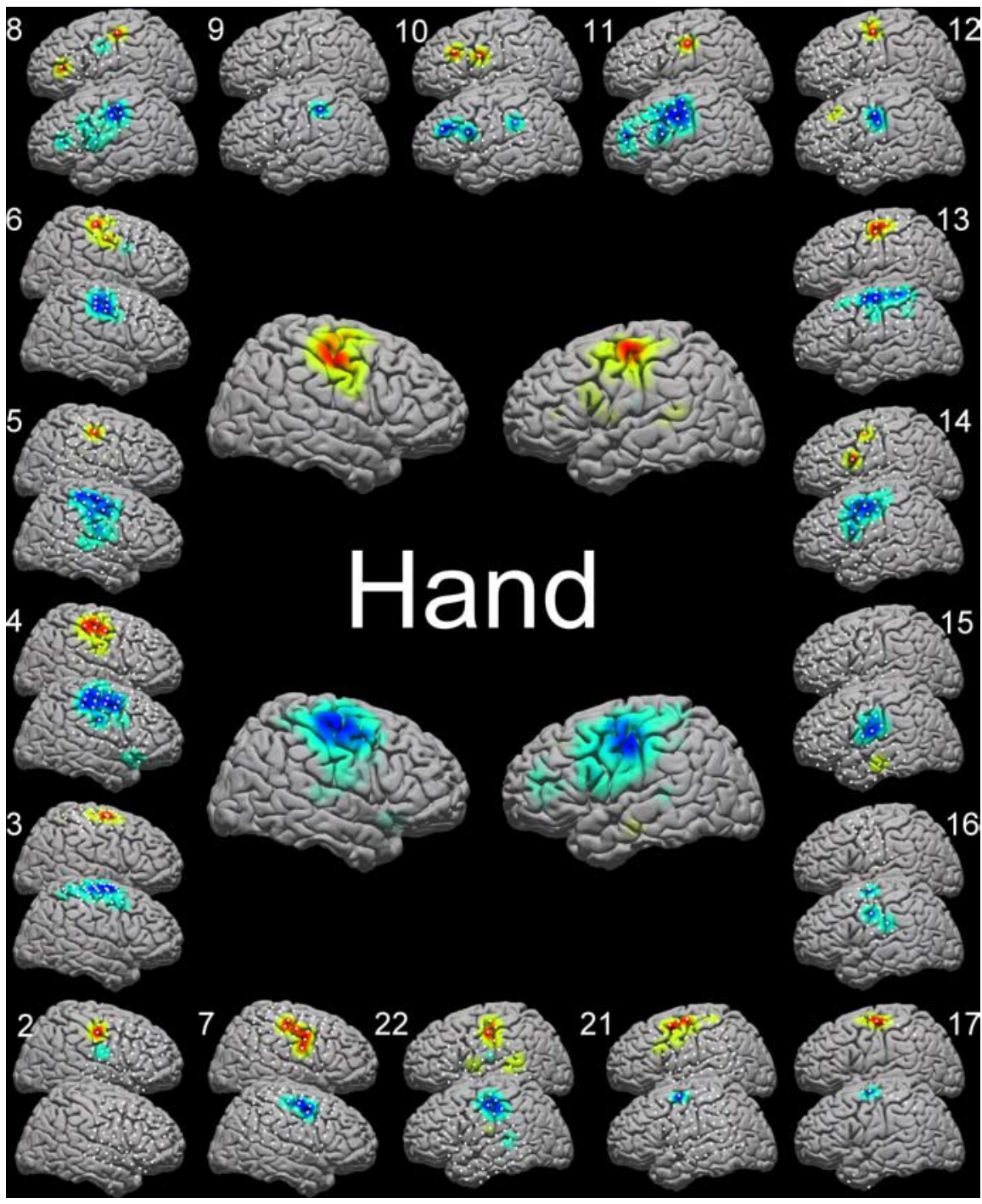

Figure 5. Cortical activation maps for hand and tongue movements. Electrode locations are shown in white. The top cortex of each pair is the HFB activation, and the bottom is the LFB activation. Outer brains, Individual patients are reflected by brain pairs around the outside of the figure, with the number next to the brain indicating the patient number, as detailed in Table 1. Centra brains, The average activations for hand and tongue movement for left and right cortices (right and left hand movement, depending on the patient's electrode locations).

In addition to the general changes across subjects, the localization of HFB and LFB changes corresponded with cortical stimulation mappings of motor cortex in individual patients (Fig. 9). Of the 20 electrode pairs that displayed responses to stimulation, at least one of the electrodes in the stimulated pair had a significant change in either the LFB ( 19 of 20 pairs) or the HFB ( 17 of 20 pairs), and thus 16 of the 20 pairs had significant changes in both the HFB and the LFB.

Finally, the spatial distribution for hand and tongue activations (Table 2) was broader for the LFB than for the HFB ( $p<$ 0.01 for both hand and tongue, using a paired $t$ test across patients). In other words, significantly larger areas of cortex exhibited changes in the LFB than in the HFB.

\section{Discussion}

Picoampere currents through individual membranous channels generate small changes in the local potential each time these channels open and close. Each neuron in cortex has millions of these channels. The potential changes that we measure at each 


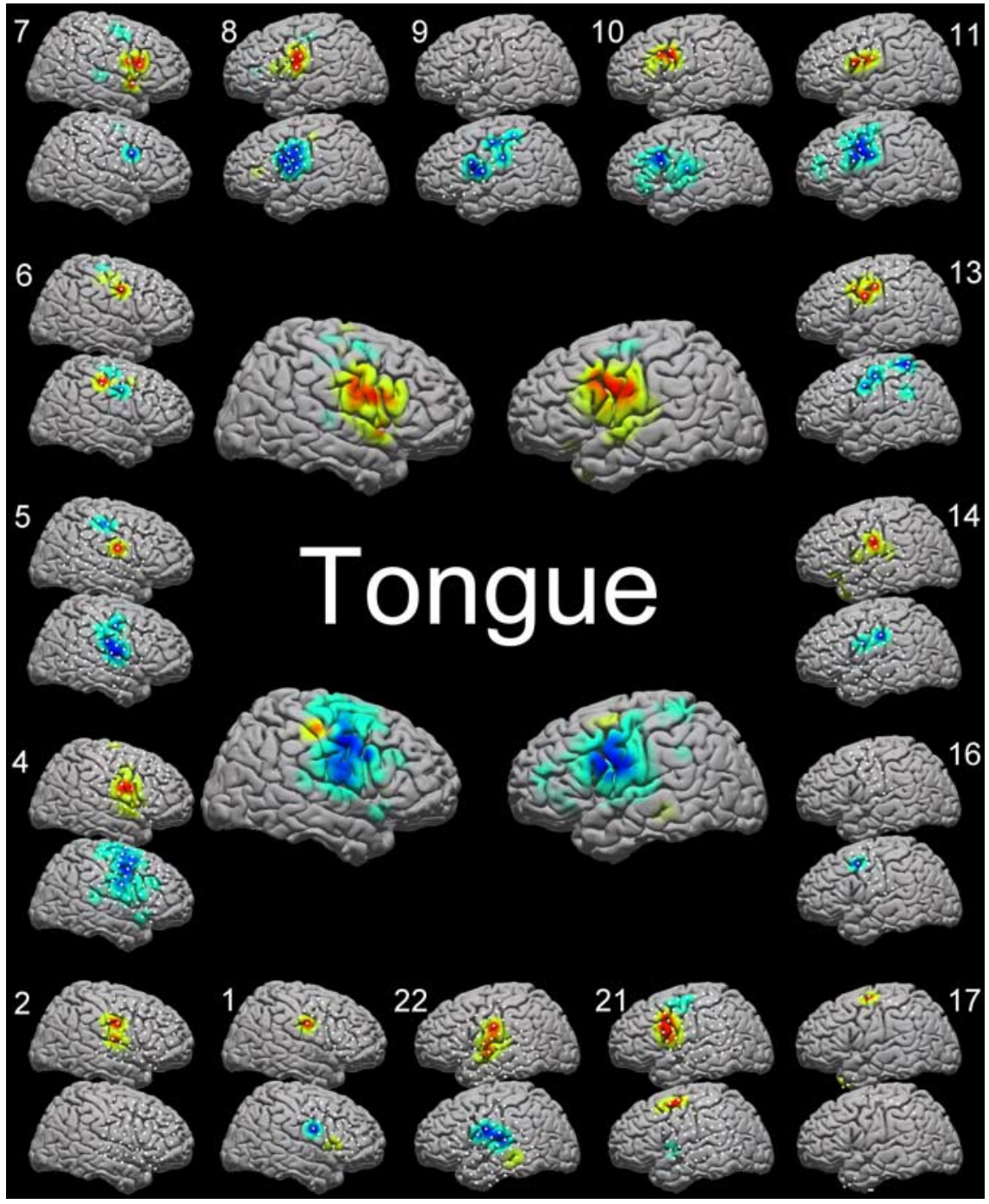

Figure 6. For details, see Figure 5. electrode of an electrocorticographic (ECoG) array on the brain surface reflect innumerable numbers of these currents. Distinct spectral shifts that are measurable, at specific frequencies, on this scale must correspond to large-scale coherence at a specific timescale (Cassidy et al., 2002; Paradiso et al., 2004), and disorganized (nonsynchronous) but global increases in electrical activity across local neuronal populations will be reflected by broad spectral increases (Surya, 1999). This study captured both of these types of changes to functionally map the cortex during a repetitive movement task.

To date, ECoG data has been analyzed on an individual subject-by-subject basis, partially because of a lack of methods for standardizing and comparing data across subjects and partially because of small study sizes. The method described in this study facilitates collation of results across subjects according to stereotactically defined anatomy. Using the present method and data collected from 20 patients, this study provides an in-depth characterization of the spectral changes in the ECoG that accompany movement of the hand or the tongue. We find, across numerous subjects, that the high-frequency band power changes are spatially focal with a broad-frequency power increase with motor activation, whereas the low-frequency band is spatially broad with more narrow bands of frequency power decrement. Like Crone et al. (1998) (Crone, 2000), our study demonstrates variable somatotopy across individuals. ERD, or spectral power reduction, was found ubiquitously at low frequency in specific peaks within the LFB,
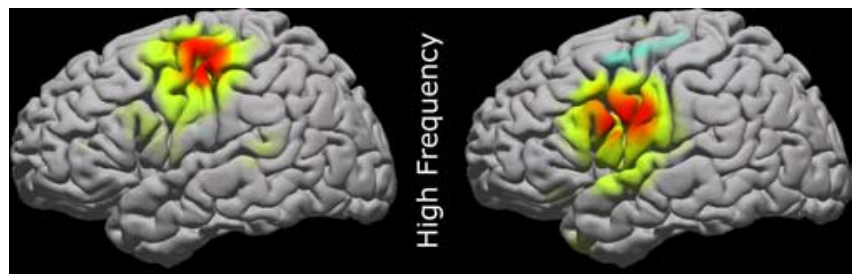

Hand Movement
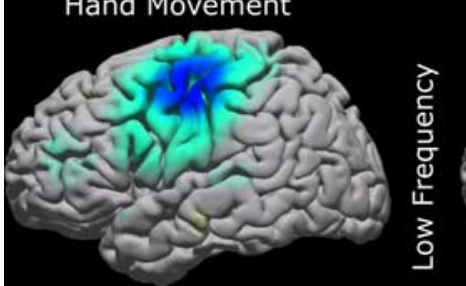

Tongue Movement

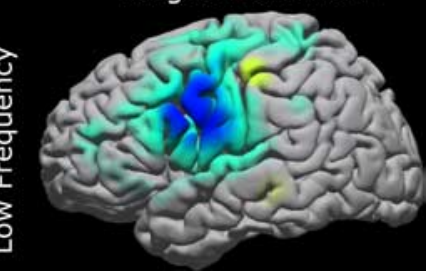

Figure 7. Average representation with contralateral electrode locations mirrored to one side (left brain). Each is scaled for the maximum increase or decrease. The somatotopic distribution of the activations is evident comparing the hand (left) and tongue (right). Additionally, there is qualitatively less spatial overlap between the HFB representations (top) than the LFB (bottom). The qualitative properties of each type of activation are detailed in Table 2. Please see the supplemental discussion for more depth about types of interpolation across individuals and why this particular technique was chosen. as described by Pfurtscheller (1999). As both Crone and Pfurtscheller have described, the high-frequency change with movement was more focal than low-frequency change, and characteristically fewer significant electrodes were detected for HFB (Table 2). We found high-frequency spectral increase to be a much more focal phenomenon than low-frequency ERD, and we found this high-frequency change to be particularly specific to rolandic cortex, across a large number of individuals. Additionally, the spectral power increase was much broader than that found in the lower-frequency bands (supplemental Fig. S4, available at www.jneurosci.org as supplemental material), and the results would have been very much the same had we picked a higher $25 \mathrm{~Hz}$ band within this broad spectral increase (supplemental Fig. S6, available at www.jneurosci.org as supplemental material).

Although there was spatial variability in the distribution of spectral changes across individuals and specific homuncular organization (more in the LFB than the HFB), there was not as much variability as that found by smaller studies. In particular, we did not find significant temporal lobe involvement, in contrast with other studies (Crone et al., 1998). Our findings do reaffirm that a rigid segmenting of motor strip somatotopy is not reflected in all aspects of the somatotopy of the electrocortico- 
graphic signal. As a particularly striking example, we found, as have Crone's studies, that lateral cortical change during a foot movement task (Fig. 8, supplemental Fig. S2, available at www.jneurosci.org as supplemental material), well outside the interhemispheric somatotopic location of stimulation and lesion effects for distal lower extremity motor cortex. Although sensorimotor activation was a consistent feature of the study across the entire cohort, the occasional single subject outlier (as in Fig. 5, subjects 8 and 10; Fig. 6, subject 6) might reflect task-related regional activation/deactivation patterns (Neuper et al., 2006) or higherorder cognitive processes.

Our method of accumulating results for different subjects does have some limitations that are worth noting. Variable electrode placement across individuals can confound interpretation when activations are considered when comparing subjects with nonoverlapping or peripheral electrode array coverage. Conversely, because the activation on the template is the average of activation ("A")-weighted Gaussian kernels, the peaks of activation will be somewhat blunted by similar sites with increased noise or reduced signal. Because the singular electrode activations are smeared to a template, this also could lead to the possibility that two independent
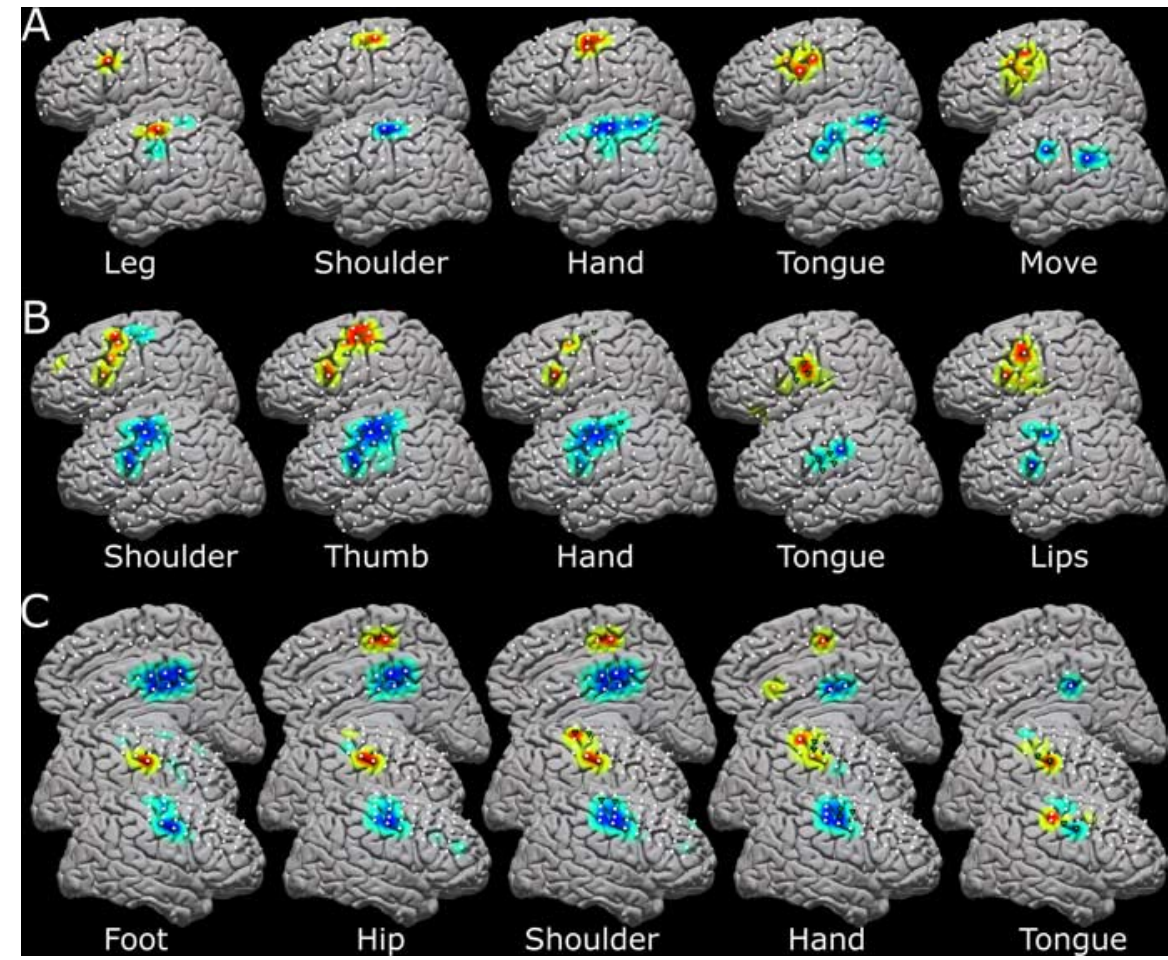

Figure 8. Five different movement modalities within the same patient for three different patients, with each relevant modality labeled. The top brains are high-frequency band representation, and the bottom are low-frequency representation. White dots indicate electrode positions, and the green triangles reflect the pairwise stimulation sites, which elicited movement of the concerned modality. $\boldsymbol{A}-\boldsymbol{C}$, Data for subjects $9(\boldsymbol{A}), 3(\boldsymbol{B})$, and $16(\boldsymbol{C})$ are shown. The medial representations in $\boldsymbol{C}$ reflect activation as reflected by a $2 \times 8$ interhemispheric strip, and the activations seen are likely supplementary motor areas rather than sensorimotor cortex.

sites of activation are grouped together.

This is especially worth considering when electrode spacing is 1 $\mathrm{cm}$ apart. Despite these limitations, our present method still allows for the development of normalized and summated electrocorticographic data from which consistent, significant, and generalized statements of motor cortex activation have been made similar to that of functional magnetic resonance imaging (fMRI). Thus far, this has been elusive in EcoG-related literature.

The topographical activations for hand and tongue displayed significant concurrence to known topography of hand and tongue motor cortices. Figures 5 and 6 illustrate the activations resulting from hand and tongue movement across 17 and 13 patients, respectively. The locations, when averaged, line up with the expected peri-rolandic sensorimotor cortical sites. It is not surprising that the activations span the central sulcus, because the task involves both motor movement and sensory feedback. Taken individually, the cortical activations approximate the homunculus, with some extrarolandic involvement. It is important to note that some of the activation maps in Figures 5 and 6 are limited by electrode coverage and that this limitation must be considered when scrutinizing the activation maps. The most dramatic example of this is subject 17, in Figures 5 and 6, in which a single strip passes through classic hand motor cortex. In Figure 5, hand activation appropriately spans two electrodes in hand area; in Figure 6 , however, tongue activation is present in the HFB in one electrode, and without any other rolandic coverage, it is not clear whether a very small HFB increase is forced to the maximum or not. This forcing of small but significant changes in peripheral areas in which there is no coverage of the primary area might help explain some of the strange findings with leg, foot, and shoulder movements (Fig. 8, row A; also several findings in supplemental
Fig. S2, available at www.jneurosci.org as supplemental material). Electrical stimulation of the cortex to create transient lesions or to induce overt movements, extraoperatively or intraoperatively, is the established gold-standard method to functionally localize motor cortex in the human brain (Ojemann et al., 1989; Chitoku et al., 2001; Branco et al., 2003). The present method contrasts with the classical stimulation method for functional localization because the method detailed here measures natural cortical change in response to functional behavioral change. Stimulation mapping, however, perturbs the natural state of the brain to nonselectively elicit functional behavioral change. Nevertheless, after pooling the HFB and LFB, electrocorticographic changes were universally seen at sites responsive to stimulation (Leuthardt et al., 2007).

Our results support the notion that high- and low-frequency band changes arise from different physiological phenomena. Low-frequency changes are postulated to arise in broad cortical areas, which are collectively regulated by central structures (e.g., the thalamus and basal ganglia). In this view, this regulation is reflected by specific, phase-coherent ("synchronized") native timescales ranging from 30 to $120 \mathrm{~ms}$ and seen as corresponding peaks in the power spectrum at $\sim 8-32 \mathrm{~Hz}$. In some subjects, this spectral "peak" was found to be exaggerated as a dynamic component associated with the task (see supplemental Fig. S5, available at www.jneurosci.org as supplemental material), although it is a feature localized to nonmovement epochs of the task, supporting the hypothesis that it is reflecting a regulatory process. That we found this is not surprising, because a rebound in power in EEG $\beta$ after movement, beyond resting power, has been well documented by Neuper and Pfurtscheller (1996) (Pfurtscheller et 


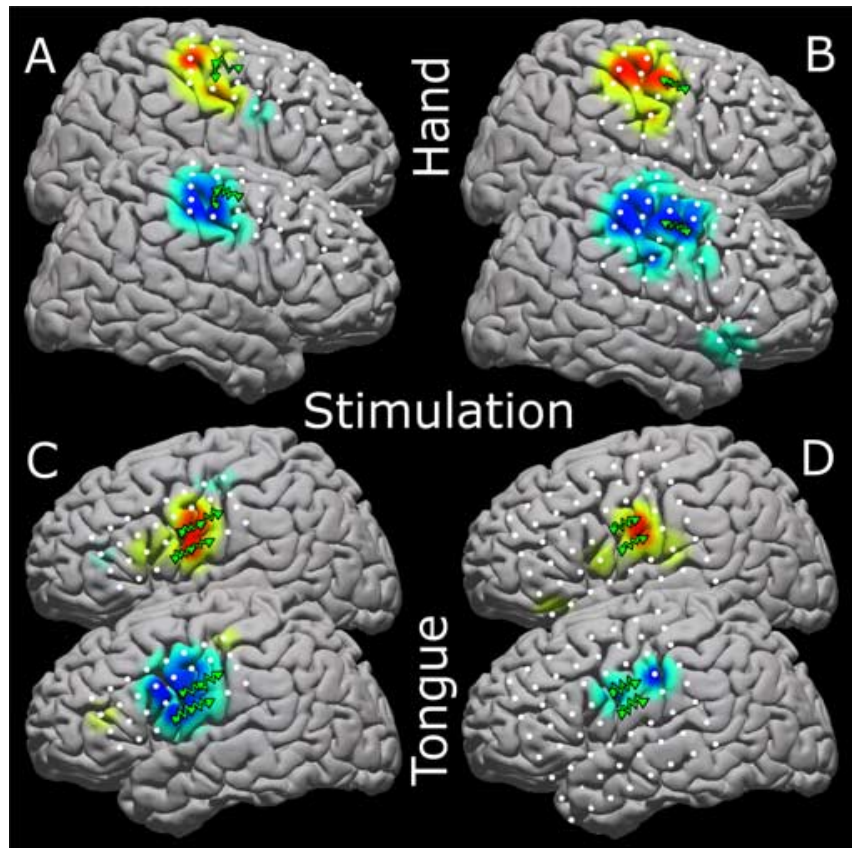

Figure 9. Four magnified brain maps to show stimulation resulting in motor movement along with the activation for the same modality. Stimulation is done pairwise; thus, elicited motor movement may be attributable to cortex beneath only one of the electrode pair. Positive electrode stimulation locations are shown with green triangles connected by jagged green lines. The top cortex of each pair is the high-frequency representation, and the bottom is the low-frequency representation. $A, B$, Hand movements with stimulation are shown for patients $16(\boldsymbol{A})$ and $6(\boldsymbol{B}) . \boldsymbol{C}, \boldsymbol{D}$, Tongue movements are shown for patients $1(\boldsymbol{C})$ and $3(\boldsymbol{D})$. Please see the supplemental discussion (available at www.jneurosci.org as supplemental material) regarding the stimulation process.

\section{Table 2. Parameters associated with all hand and tongue movement findings}

\begin{tabular}{lcc}
\hline Motor modality & Hand & Tongue \\
\hline Total electrodes & 892 & 764 \\
Number of high band electrodes & 75 & 85 \\
Mean high band y position & $-15 \pm 11$ & $-6 \pm 13$ \\
Mean high band z position & $50 \pm 11$ & $31 \pm 19$ \\
Number of low band electrodes & 172 & 129 \\
Mean low band $y$ position & $-15 \pm 14$ & $-6 \pm 14$ \\
Mean low band z position & $50 \pm 14$ & $39 \pm 18$ \\
\hline
\end{tabular}

The position values refer to Talairach standardized coordinates (Talairach and Tournoux, 1988) (in $\mathrm{mm}$ ), and the $x$ coordinates are omitted because data from the lateral surface of both hemispheres has been combined. The \pm symbol denotes SD in position (square-root of the variance).

al., 1998). Also, monkey LFP experiments have shown load-based increases in this peak during rest periods a gripping task with variable resistance, suggesting that it is dynamically related to movement task properties (Jackson et al., 2002). Recent studies comparing this movement-associated $\beta$ rebound in subjects with and without ventrolateral thalamotomy have demonstrated a role for the intact thalamus in the dynamic nature of the $\beta$ rhythm (Van Der Werf et al., 2006). Other studies involving both modeling (Destexhe et al., 1998) and experiment indicated the importance of both the thalamus (Paradiso et al., 2004) and basal ganglial structures (Cassidy et al., 2002; Foffani et al., 2005) in $\beta$ modulation in humans. Beginning at $40-50 \mathrm{~Hz}$, a broad taskdependent increase in spectral power is measured reliably across patients and movement modalities (Figs. 1, 2, 4, supplemental Fig. S4, available at www.jneurosci.org as supplemental material). It has been suggested that this spectral power increase at these higher frequencies is correlated with increased blood oxygenation level-dependent signal in fMRI studies (Mukamel et al.,
2005), that (as we demonstrate here) high-frequency changes are a specific correlate of local behavior (Hoogenboom et al., 2006; Womelsdorf et al., 2006), and we postulate that they may directly reflect an increase in local cortical computation. Thus, it is possible that the observed high-frequency effect is caused by the integrated activity of firing rates of cells immediately underneath the electrodes. That is, a global shift in the power law noise spectra (Surya, 1999), accompanying an increase in local, corticocortical activity [as observed in LFPs (Beggs and Plenz, 2003)], would be also present at low frequencies. However, observation of this shift at low frequencies could be masked by the influence of thalamocortical and basal ganglial-cortical interactions reflected at discrete timescales in the low-frequency spectral region. This implies that the notions of synchronization and desynchronization might not be applicable to these changes and that the effect of increase in band power might actually extend across all frequencies including those in the $\mu / \beta$ range.

\section{Conclusion}

Electrocorticography can provide the basis for functional maps that reveal motor cortical organization in an efficient and reproducible manner. Using simple localization methods and spectral analyses, we derived robust motor activations, consistent across individuals and concurrent with parallel cortical stimulation maps. High-frequency spectral power increased with activity, was spectrally broad, and may reflect activity of local neuronal populations. The increases in activity seem to conform to exponential or power law noise behavior with shifts in the critical exponent that correlate with behavior, rather than demonstrating increases at specific frequencies. Low-frequency spectral power decreased with activity, was spectrally focused narrowly over traditional $\mu / \beta$-frequency ranges, and thus may reflect the dissolution of spectral peaks, which may themselves reflect the regulatory timescales involved with the interaction between central brain structures and the cortical surface (i.e., thalamocortical and basal ganglia-cortical). We quantified and mapped these effects across a large number of patients, showing the spatial distribution of the low-frequency (regulatory) band to be spatially broader than the high-frequency (activity-based) band. The results of the topographical mapping reveal somatotopic representation of these signals, which typically follow the traditional homunculus model. In summary, our results provide, for the first time, an in-depth characterization of spectral changes in ECoG signals during movement across a reasonably large number of subjects; the techniques presented in this study provide a basis for electrocorticography-based, quantitative mapping of motor modalities in the human brain.

\section{References}

Beggs JM, Plenz D (2003) Neuronal avalanches in neocortical circuits. J Neurosci 23:11167-11177.

Beggs JM, Plenz D (2004) Neuronal avalanches are diverse and precise activity patterns that are stable for many hours in cortical slice cultures. J Neurosci 24:5216-5229.

Berger H (1929) Über das Elektroenkephalogramm des Menschen. Arch Psychiatr Nervenkr 87:527-570.

Bonferroni C (1936) Teoria statistica delle classi e calcolo delle probabilità. Pubblicazioni del R Istituto Superiore di Scienze Economiche e Commerciali di Firenze. 8:3-62.

Branco DM, Coelho TM, Branco BM, Schmidt L, Calcagnotto ME, Portuguez M, Neto EP, Paglioli E, Palmini A, Lima JV, Da Costa JC (2003) Functional variability of the human cortical motor map: electrical stimulation findings in perirolandic epilepsy surgery. J Clin Neurophysiol 20:17-25. Brazier MA (1961) Recordings from large electrodes. Methods Med Res 9:405-432. 
Cassidy M, Mazzone P, Oliviero A, Insola A, Tonali P, Di Lazzaro V, Brown P (2002) Movement-related changes in synchronization in the human basal ganglia. Brain 125:1235-1246.

Chitoku S, Otsubo H, Harada Y, Jay V, Rutka JT, Weiss SK, Abdoll M, Snead III OC (2001) Extraoperative cortical stimulation of motor function in children. Pediatr Neurol 24:344-350.

Collins DH, Holmes CJ, Peters TM, Evans AC (1995) Automatic 3-D model-based neuroanatomical segmentation. Hum Brain Mapp 3:190-208.

Crone NE (2000) Functional mapping with ECoG spectral analysis. Adv Neurol 84:343-351.

Crone NE, Miglioretti DL, Gordon B, Sieracki JM, Wilson MT, Uematsu S, Lesser RP (1998a) Functional mapping of human sensorimotor cortex with electrocorticographic spectral analysis. I. Alpha and beta eventrelated desynchronization. Brain 121:2271-2299.

Crone NE, Miglioretti DL, Gordon B, Lesser RP (1998b) Functional mapping of human sensorimotor cortex with electrocorticographic spectral analysis. II. Event-related synchronization in the gamma band. Brain 121: 2301-2315.

Destexhe A, Contreras D, Steriade M (1998) Mechanisms underlying the synchronizing action of corticothalamic feedback through inhibition of thalamic relay cells. J Neurophysiol 79:999-1016.

Foffani G, Bianchi AM, Baselli G, Priori A (2005) Movement-related frequency modulation of beta oscillatory activity in the human subthalamic nucleus. J Physiol 568:699-711.

Fox PT, Perlmutter JS, Raichle ME (1985) A stereotactic method of anatomical localization for positron emission tomography. J Comput Assist Tomogr 9:141-153.

Hoogenboom N, Schoffelen JM, Oostenveld R, Parkes LM, Fries P (2006) Localizing human visual gamma-band activity in frequency, time and space. NeuroImage 29:764-773.

Hoshida T, Sakaki T (2003) Functional brain mapping detected by cortical stimulation using chronically implanted subdural electrodes: basic knowledge of clinical nerve physiology for neurosurgeons. No Shinkei Geka 31:811-819.

Jackson A, Spinks RL, Freeman TC, Wolpert DM, Lemon RN (2002) Rhythm generation in monkey motor cortex explored using pyramidal tract stimulation. J Physiol 541:685-699.

Kostov A, Polak M (2000) Parallel man-machine training in development of EEG-based cursor control. IEEE Trans Rehabil Eng 8:203-205.

Leuthardt EC, Schalk G, Wolpaw JR, Ojemann JG, Moran DW (2004) A brain-computer interface using electrocorticographic signals in humans. J Neural Eng 1:63-71.

Leuthardt EC, Miller KJ, Schalk G, Rao RP, Ojemann JG (2006) Electrocorticography-based brain computer interface-the Seattle experience. IEEE Trans Neural Syst Rehabil Eng 14:194-198.

Leuthardt M, Anderson, Schalk, Dowling, Moran, Ojemann, (2007) Electrocorticographic frequency alteration mapping (EFAM), a novel clinical technique for mapping motor cortex. Neurosurgery, in press.

Levine SP, Huggins JE, BeMent SL, Kushwaha RK, Schuh LA, Passaro EA, Rohde MM, Ross DA (1999) Identification of electrocorticogram patterns as the basis for a direct brain interface. J Clin Neurophysiol 16:439-447.

Lopes da Silva FH (1991) Neural mechanisms underlying brain waves: from neural membranes to networks. Electroenceph Clin Neurophysiol 79:81-93.

Marsden JF, Werhahn KJ, Ashby P, Rothwell J, Noachtar S, Brown P (2000) Organization of cortical activities related to movement in humans. J Neurosci 20:2307-2314

Miller KJ, Makeig S, Hebb AO, Rao RPN, denNijs M, Ojemann JG (2007) Cortical electrode localization from $\mathrm{x}$-ray and simple mapping for electrocorticographic research: the "location on cortex" (LOC) package for Matlab. J Neurosci Methods, in press.

Modayur B, Prothero J, Ojemann G, Maravilla K, Brinkley J (1997) Visualization-based mapping of language function in the brain. NeuroImage 6:245-258.

Mukamel R, Gelbard H, Arieli A, Hasson U, Fried I, Malach R (2005) Coupling between neuronal firing, field potentials, and FMRI in human auditory cortex. Science 309:951-954.
Neidermeyer E (1999) Electroencephalography: basic principles, clinical applications and related fields (Niedermeyer E, Lopes da Silva FH, eds), 149-173. Baltimore: Williams and Wilkins.

Neuper C, Pfurtscheller G (1996) Post-movement synchronization of beta rhythms in the EEG over the cortical foot area in man. Neurosci Lett 216:17-20.

Neuper C, Wortz M, Pfurtscheller G (2006) ERD/ERS patterns reflecting sensorimotor activation and deactivation. Prog Brain Res 159:211-222.

Nunez PL, Wingeier BM, Silberstein RB (2001) Spatial-temporal structures of human alpha rhythms: theory, microcurrent sources, multiscale measurements, and global binding of local networks. Hum Brain Mapp 13:125-164.

Nuttall A (1981) Some windows with very good sidelobe behavior. IEEE Trans Acoust 29:84-91.

Ojemann GA (1982) Models of the brain organization for higher integrative functions derived with electrical stimulation techniques. Hum Neurobiol $1: 243-249$.

Ojemann G, Ojemann J, Lettich E, Berger M (1989) Cortical language localization in left, dominant hemisphere. An electrical stimulation mapping investigation in 117 patients. J Neurosurg 71:316-326.

Paradiso G, Cunic D, Saint-Cyr JA, Hoque T, Lozano AM, Lang AE, Chen R (2004) Involvement of human thalamus in the preparation of self-paced movement. Brain 127:2717-2731.

Pfurtscheller G (1981) Central beta rhythm during sensorimotor activities in man. Electroencephalogr Clin Neurophysiol 51:253-264.

Pfurtscheller G (1999) Event-related desynchronization (ERD) and eventrelated synchronization (ERS). In: Electroencephalography: basic principles, clinical applications, and related fields (Neidermeyer E, Lopes da Silva F, eds), pp 958-967. Baltimore: Williams and Wilkins.

Pfurtscheller G (2000) Spatiotemporal ERD/ERS patterns during voluntary movement and motor imagery. Suppl Clin Neurophysiol 53:196-198.

Pfurtscheller G (2001) Functional brain imaging based on ERD/ERS. Vision Res 41:1257-1260.

Pfurtscheller G, Zalaudek K, Neuper C (1998) Event-related beta synchronization after wrist, finger and thumb movement. Electroencephalogr Clin Neurophysiol 109:154-160.

Pfurtscheller G, Graimann B, Huggins JE, Levine SP, Schuh LA (2003) Spatiotemporal patterns of beta desynchronization and gamma synchronization in corticographic data during self-paced movement. Clin Neurophysiol 114:1226-1236.

Pfurtscheller G, Brunner C, Schlogl A, Lopes da Silva FH (2006) Mu rhythm (de)synchronization and EEG single-trial classification of different motor imagery tasks. NeuroImage 31:153-159.

Rohde MM, BeMent SL, Huggins JE, Levine SP, Kushwaha RK, Schuh LA (2002) Quality estimation of subdurally recorded, event-related potentials based on signal-to-noise ratio. IEEE Trans Biomed Eng 49:31-40.

Schalk G, McFarland DJ, Hinterberger T, Birbaumer N, Wolpaw JR (2004) BCI2000: a general-purpose brain-computer interface (BCI) system. IEEE Trans Biomed Eng 51:1034-1043.

Surya C, ed (1999) Proceedings of the 15th International Conference on noise in Physical Systems and 1/f Fluctuations. London: Bentham.

Talairach J, Tournoux P (1988) Talairach: co-planar stereotaxic atlas of the human brain. Stuttgart, Germany: Thieme.

Van Der Werf YD, Sadikot AF, Strafella AP, Paus T (2006) The neural response to transcranial magnetic stimulation of the human motor cortex. II. Thalamocortical contributions. Exp Brain Res 175:246-255.

Wolpaw JR, McFarland DJ, Neat GW, Forneris CA (1991) An EEG-based brain-computer interface for cursor control. Electroencephalogr Clin Neurophysiol 78:252-259.

Womelsdorf T, Fries P, Mitra PP, Desimone R (2006) Gamma-band synchronization in visual cortex predicts speed of change detection. Nature 439:733-736.

Zacks JM, Gilliam F, Ojemann JG (2003) Selective disturbance of mental rotation by cortical stimulation. Neuropsychologia 41:1659-1667.

Zacks JM, Michelon P, Vettel JM, Ojemann JG (2004) Functional reorganization of spatial transformations after a parietal lesion. Neurology 63: 287-292. 